\title{
Vulnerability Analysis of Urban Natural Gas Network under Cascading
}

\section{Failures}

\author{
Y.Wang, H.Huang*, B.N. Su \\ Institute of Public Safety Research, Department of Engineering Physics, Tsinghua University, Beijing, China
}

\begin{abstract}
In this paper, a new vulnerability metric for urban natural gas network under cascading failures are proposed, which is based on hydraulic analysis method. Simulation result of a simplified network shows that pressure-driven hydraulic method is more realistic than demand-driven hydraulic method because user's demand is not fully satisfied under a cascading failure situation. Then the vulnerability analysis procedure is applied to a real-world natural gas network and vulnerability maps under different source assumptions are acquired. The result shows that the spatial distribution of network vulnerability depends heavily on the location of natural gas sources.
\end{abstract}

KEYWORD: Vulnerability analysis; Natural gas network; Cascading failures; Risk management

\section{INTRODUCTION}

In recent years, research of cascading failures in various critical infrastructure networks has been a hot area (Dai, Y. et al. 2014, Shuang, Q. et al. 2014). Here the word cascading failure refers to the phenomenon where failure of some parts in a network system causes the redistribution of network load and flow, thus causing the failure of more parts. As is known to all, urban natural gas network is one of the critical infrastructures, on which the stable operation of modern cities strongly depend. Once natural gas network suffers a terrorist attack or other severe destructions like an earthquake, there will be a huge loss due to cascading failure. So, studying the vulnerability of urban gas network from the perspective of cascading failure is of great and practical significance to the safe operation and planning of cities and towns.

\section{HYDRAULIC ANALYSIS OF NATURAL GAS NETWORK}

\subsection{Topology extraction}

Natural gas networks are usually complex systems mainly consisting of gas distributing stations, compressor stations, regulator devices, pipelines of different stress levels and gas storages. When it comes to those zones near consumers, most pipelines are low-pressure and there are almost no regulator devices. In order to implement the hydraulic analysis method, the topology of natural gas network needs to be expressed as a connection matrix (Han, Z. Y. \& Weng, W. G. 2010.). Pipelines are represented by edges and the junctions of pipelines including gas sources and consumers are represented by nodes. Connection matrix A is defined as:

$A(i, j)=\left\{\begin{array}{c}1, \text { node } i \text { is the end of pipeline } j \\ -1, \text { node } i \text { is the start of pipeline } j \\ 0, \text { else }\end{array}\right.$

\subsection{Basic equations}

(1) Continuity equation

$$
A Q=q
$$

where $\mathrm{Q}$ is the pipeline flow $\left(\mathrm{m}^{3} / \mathrm{h}\right), \mathrm{q}$ is the node flow $\left(\mathrm{m}^{3} / \mathrm{h}\right)$.

(2) Pipeline pressure drop equation

$$
A^{T} p=\Delta P
$$

where $\mathrm{p}$ is the node relative pressure $(\mathrm{Pa}), \Delta \mathrm{P}$ is the pressure drop of pipelines $(\mathrm{Pa})$.

\section{(3) Admittance matrix}

$Y=A G A^{\prime}$

where $G$ is the inverse matrix of $S^{\prime}$, for lowpressure pipelines $S^{\prime}$ is defined as:

$$
S^{\prime}(j, j)=6.26 \times 10^{7} \lambda \frac{Q(j)}{d(j)^{5}} \rho \frac{T}{T_{0}} l(j)
$$


where $\lambda$ is frictional resistance coefficient which depends on the reynolds number, $\mathrm{d}(\mathrm{j})$ is the diameter of pipeline $\mathrm{j}(\mathrm{mm}), \rho$ is the density of natural gas $\left(\mathrm{kg} / \mathrm{m}^{3}\right), \mathrm{T}$ is the temperature inside pipelines $(\mathrm{K})$, $\mathrm{T}_{0}=273.15 \mathrm{~K}$.

According to the basic principle of hydraulic analysis, the following equations are given:

$Q=G \Delta P$

$Y p=q$

\subsection{Algorithm flowchart}

The main procedure of hydraulic analysis is displayed in Figure 1. The required input includes node flow q, pipe diameter d, pipe length 1 and initial pipe flow Q. When the computational accuracy of pipe flow Q is satisfied, the steady flow state under node flow $\mathrm{q}$ is acquired, which includes node pressure $\mathrm{p}$. It is obvious that both pipeline diameter $\mathrm{d}$ and pipeline length 1 do not change easily, and initial pipe flow doesn't affect the final steady state. What matters is node flow q.

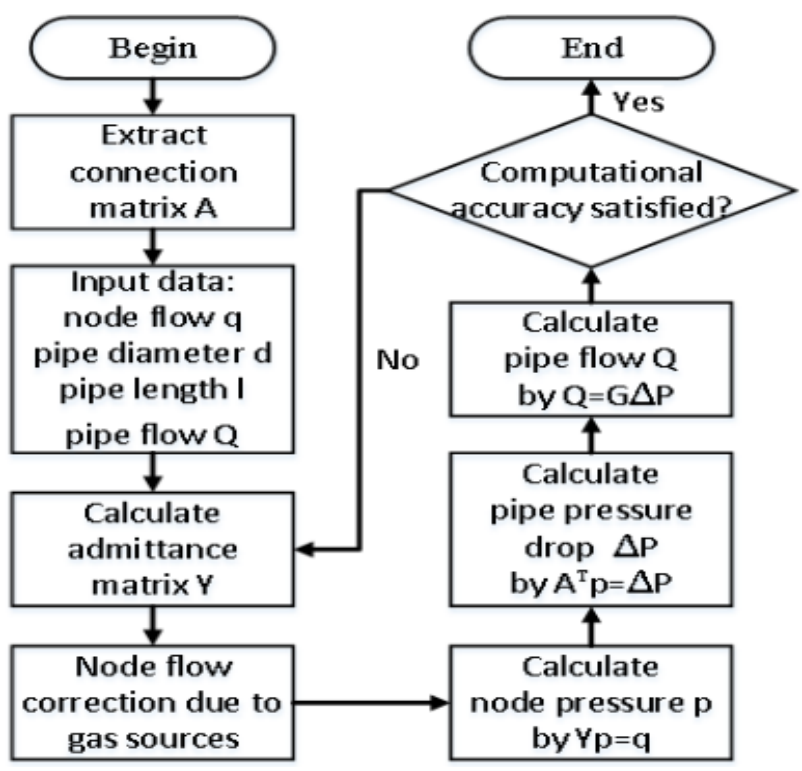

Figure 1. Flowchart for hydraulic analysis.

\subsection{Application to a simplified network}

In order to verify the validity and correct implementation of hydraulic analysis method, the hydraulic state of a simplified natural gas network with fourteen nodes and twenty pipelines is analyzed (Li, C. M. 1997.). Simplified network in the rest of this paper all refers to this one. Figure 2 includes the network topology and all the required parameters for the calculation, including source information, node flow, pipeline diameter, and pipeline length. Figure 3 shows that the result is consistent with that in Zhao's work (Zhao, L. 2009.).

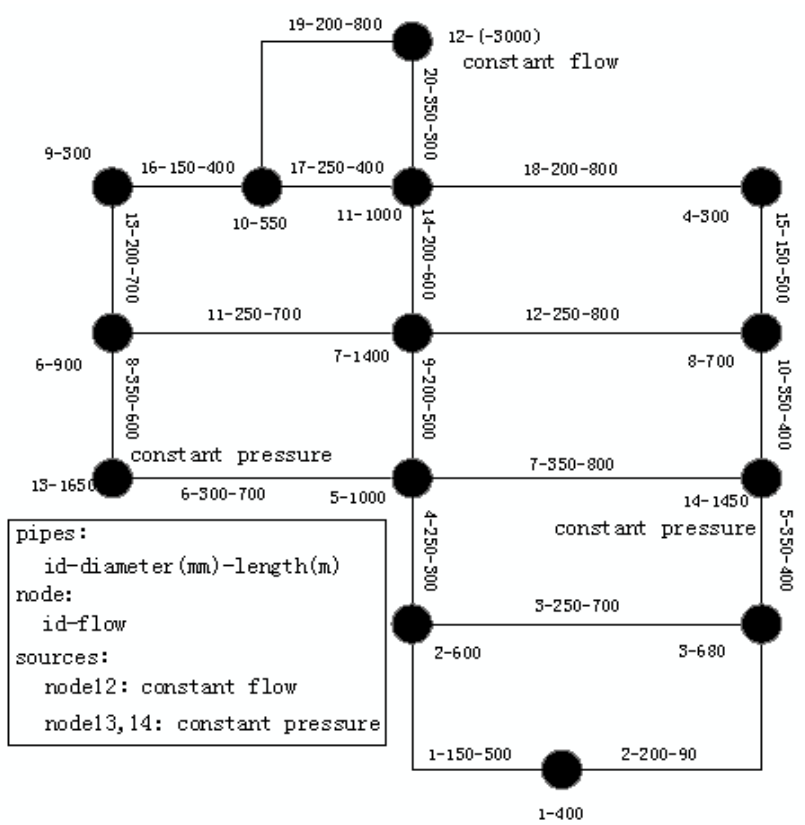

Figure 2. A simplified natural gas network

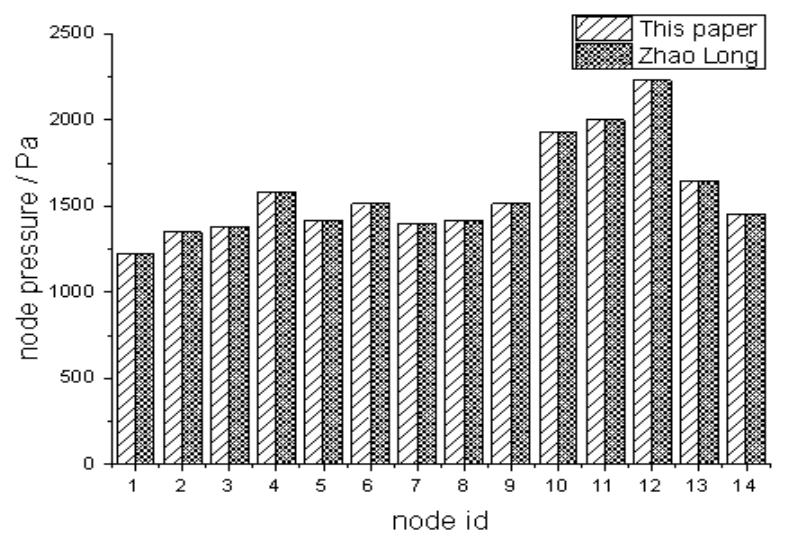

Figure 3. Node pressure calculated by hydraulic analysis

\section{VULNERABILITY ANALYSIS METHOD}

\subsection{Vulnerability metrics}

The vulnerability of natural gas network can be understood from two aspects: structural and functional. Structural vulnerability means how easily physical destruction of the network components will occur. Functional vulnerability corresponds to how easily the network will lose all or part of its function. This two concepts are closely related but not the same. This paper focuses on the latter one, namely, functional vulnerability under cascading failure.

The function of natural gas network is to deliver natural gas to customers, which requires a proper gas pressure at endpoints. So the loss of function can be interpreted as the loss of node pressure near customers.

As mentioned above, the most important input for hydraulic analysis is the node flow vector. For vulnerability analysis, the most relevant output is the node pressure. Both node leakage and pipeline leakage can be treated as an increase of several node flow nearby. Figure 4 shows an example of how the change of a node's flow affect other nodes' pressure. 
As the increase of node 4's flow, the pressure of node 10 and node 11 decrease correspondingly. The consequence of leakage at node 4 is considered as the vulnerability of the gas network at node 4 . We use the slop given by linear fitting to capture this consequence. The new vulnerability metric is defined as:

$V_{i}=\sum_{j \in G}\left|k_{i j}\right|$

where $\mathrm{V}_{\mathrm{i}}$ is the network vulnerability at node $\mathrm{i}, \mathrm{k}_{\mathrm{ij}}$ is the slope fitted by node j's pressure against node i's flow, G represents the entire network.

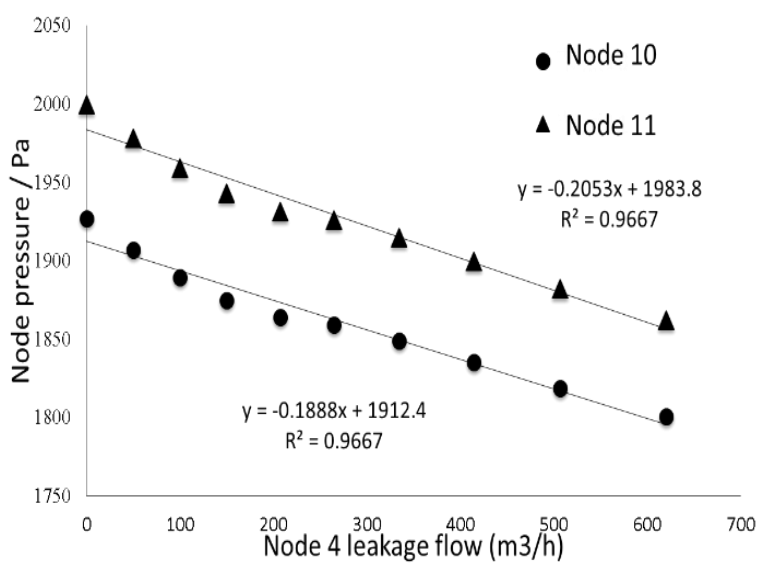

Figure 4. Influence of node 4's leakage on the pressure of node 10 and node 11 .

\subsection{Demand-driven hydraulic analysis}

Demand-driven method assumes that user's demand is still fully satisfied under cascading failure, which overestimates the node flow because the input node flow equals the sum of users' flow and leakage flow.

\subsection{Pressure-driven hydraulic analysis}

Pressure-driven method considers the effect the cascading failure has on users' flow, where users' flow is modified according to node pressure, making the result more realistic. Considering the similarity of governing equations between natural gas network and water distribution network, the Wagner model describing the actual water supplied to customers (Wagner, J. M. et al 1988) is applied, which is expressed as:

$$
q_{i, \text { user }}=\left\{\begin{array}{cl}
0 \quad, \quad p_{i} \leq p_{i, \text { min }} \\
q_{i, u s e r}^{o} \sqrt{\frac{p_{i}-p_{i, \min }}{p_{i, \text { ser }}-p_{i, \min }}}, & p_{i, \text { min }} \leq p_{i} \leq p_{i, \text { ser }} \\
q_{i, \text { user }}^{o} \quad, & p_{i} \geq p_{i, \text { ser }}
\end{array}\right.
$$

where $\mathrm{q}_{i, \text { user }}$ is the user demand actually satisfied at node $\mathrm{i}, \mathrm{q}_{\mathrm{i} \text {,user }}{ }^{0}$ is the original user demand at node $\mathrm{i}$, $\mathrm{P}_{\mathrm{i}}$ is the pressure of node $\mathrm{i}, \mathrm{P}_{\mathrm{i}, \mathrm{min}}$ is the minimum pressure below which node flow will be shut down due to safety reasons, $\mathrm{P}_{\mathrm{i}, \mathrm{ser}}$ is the pressure above which user's demand at node i can be fully satisfied.

\subsection{Calculation procedure}

The whole calculation procedure for the vulnerability analysis of natural gas network under cascading failure is displayed in Figure 5.

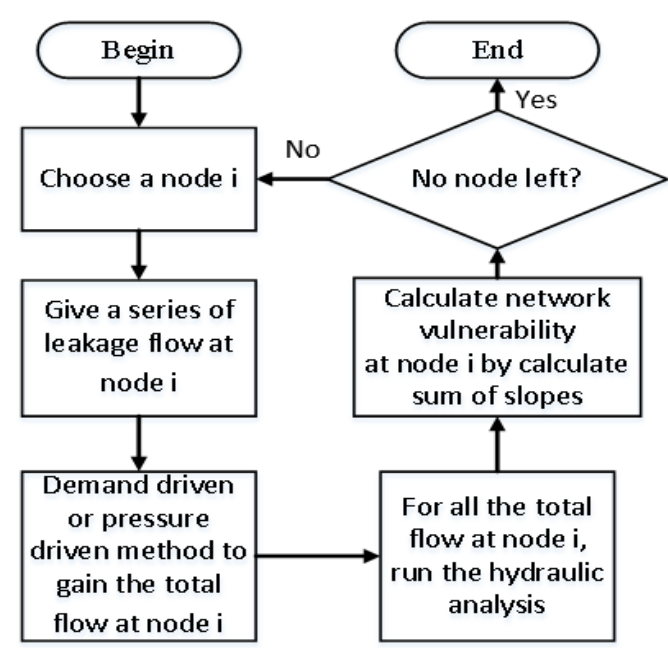

Figure 5. Flowchart for vulnerability analysis of natural gas network.

\section{APPLICATION RESULTS}

\subsection{Simplified network}

The proposed method for vulnerability analysis is first applied to a simplified network to validate its feasibility and to compare the result of demanddriven analysis and pressure-driven analysis. Figure 6 gives the vulnerability distribution by means of the two methods respectively. Demand-driven hydraulic analysis ignores the decrease of user flow, which makes nodes' total flow bigger than the real value, causing the overestimate of node vulnerability. It can be seen from Figure 6 that nodes with higher vulnerability tend to be more overestimated. This is because high vulnerable nodes are more sensitive to the change of node flow under the vulnerability definition in this paper.

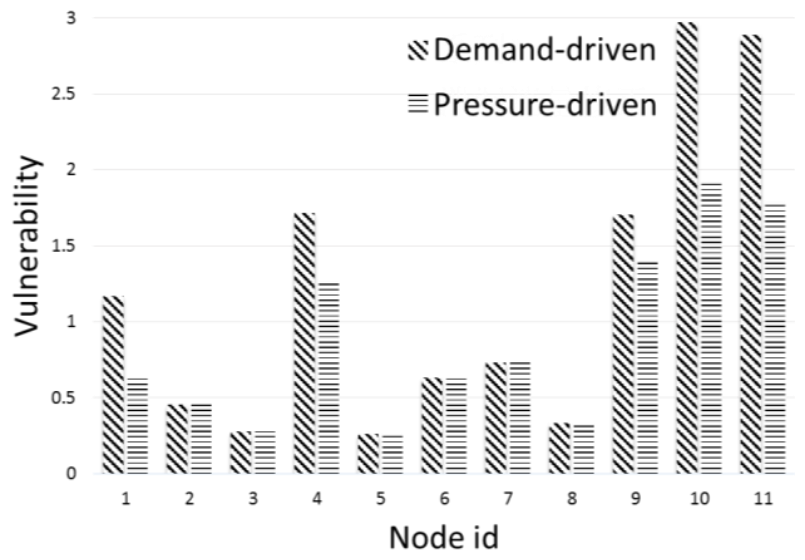

Figure 6. Vulnerability of nodes: comparison of demanddriven and pressure-driven. 


\subsection{Real-world network}

This part, the pressure-driven vulnerability analysis method is applied to a larger real-world urban natural gas network with 92 nodes and 95 pipelines.

The vulnerability definition in this paper makes the value of vulnerability depend heavily on the flow state of natural gas network before a cascading failure. Due to lack of users' demand data, it is assumed that all the users' demand are the same. Then vulnerability distribution under different source locations are calculated. In Figure 7 and Figure 8, the spatial distribution of pipeline vulnerability is displayed. The node marked by a box is the natural gas source, whose pressure is assumed to be constant.

It is clear in Figures 7-8 that the closer the pipeline is to the constant-pressure gas source, the lower the vulnerability value will be.

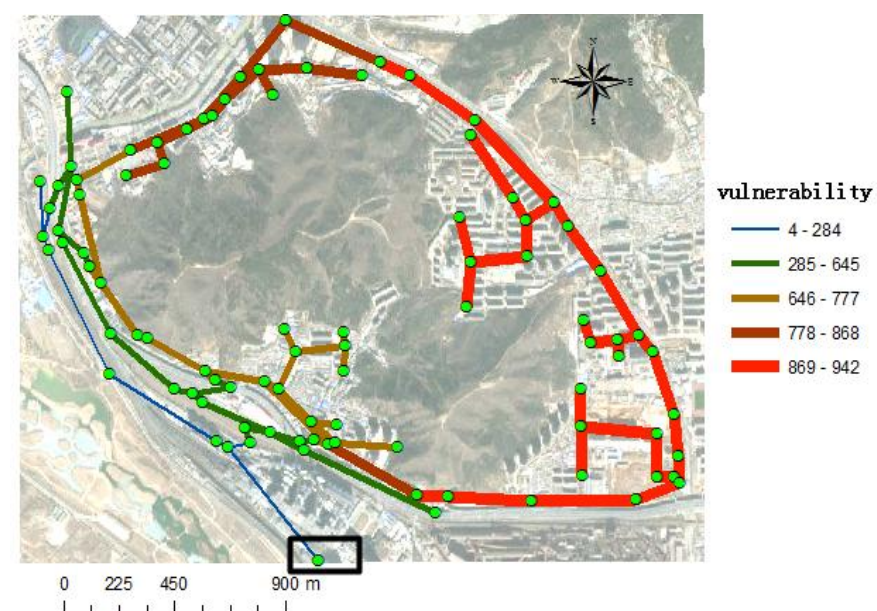

Figure 7. Vulnerability distribution under source assumption 1.

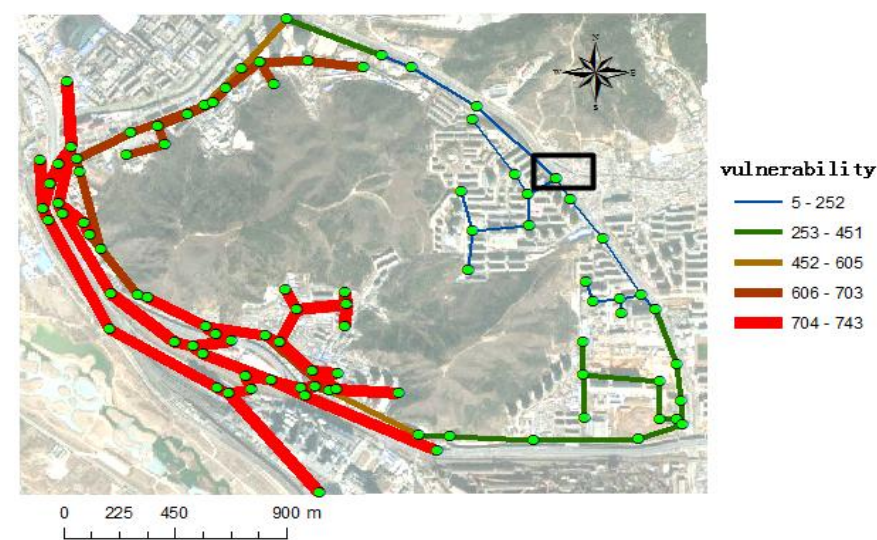

Figure 8. Vulnerability distribution under source assumption 2.

\section{CONCLUSION}

In this study, a new vulnerability metric for natural gas network is proposed. Then the difference of demand-driven hydraulic method and pressuredriven one is investigated by application to a simplified network, showing that demand-driven method overestimates the vulnerability because of its ignorance of supply drop caused by cascading failure. Finally, the pressure-driven framework is applied to a real-world natural gas network, showing that the spatial distribution of network vulnerability depends heavily on the location of natural gas sources.

\section{ACKNOWLEDGMENT}

This work was supported by the National Natural Science Foundation of China under Grant No. 71173128 and Beijing Research Center of Urban System Engineering.

\section{REFERENCES}

[1] Dai, Y. \& Chen, G. \& Dong, Z. \& Xue, Y. \& Hill, D. J. \& Zhao, Y. 2014. An improved framework for power grid vulnerability analysis considering critical system features. Physica A: Statistical Mechanics and its Applications, 395, 405-415.

[2] Han, Z. Y. \& Weng, W. G. 2010. An integrated quantitative risk analysis method for natural gas pipeline network. Journal of Loss Prevention in the Process Industries, 23(3), 428-436.

[3] Li, C. M. 1997. Design basis of gas network hydraulic calculation procedure. China Coal Industry Publishing House. (in Chinese).

[4] Shuang, Q. \& Zhang, M. \& Yuan, Y. 2014. Node vulnerability of water distribution networks under cascading failures. Reliability Engineering \& System Safety, 124, 132-141.

[5] Wagner, J. M. \& Shamir, U. \& Marks, D. H. 1988. Water distribution reliability: simulation methods. Journal of Water Resources Planning and Management, 114(3), 276294.

[6] Zhao, L. 2009. Leakage analysis and steady-state leakage simulation of natural gas network (Master's thesis, Kunming University of Science and Technology). (in Chinese) . 